\title{
TEMPORAL ANALYSIS AND AUTOMATIC CALIBRATION OF THE VELODYNE HDL-32E LIDAR SYSTEM
}

\author{
Ting On Chan ${ }^{\mathrm{a}}$, Derek D. Lichti ${ }^{\mathrm{a}}$ and David Belton ${ }^{\mathrm{b}, \mathrm{c}}$ \\ ${ }^{a}$ Department of Geomatics Engineering, University of Calgary \\ 2500 University Dr NW, Calgary, Alberta, T2N1N4 Canada \\ (ting.chan, ddlichti)@ucalgary.ca \\ ${ }^{\mathrm{b}}$ Department of Spatial Sciences, Curtin University, Perth, Australia \\ ${ }^{\mathrm{c}}$ Cooperative Research Centre for Spatial Information (CRCSI) \\ d.belton@curtin.edu.au
}

Commission V, WG V/3

KEY WORDS: LiDAR, Calibration, Segmentation, Cylindrical Feature, Accuracy

\begin{abstract}
:
At the end of the first quarter of 2012, more than 600 Velodyne LiDAR systems had been sold worldwide for various robotic and high-accuracy survey applications. The ultra-compact Velodyne HDL-32E LiDAR has become a predominant sensor for many applications that require lower sensor size/weight and cost. For high accuracy applications, cost-effective calibration methods with minimal manual intervention are always desired by users. However, the calibrations are complicated by the Velodyne LiDAR's narrow vertical field of view and the very highly time-variant nature of its measurements. In the paper, the temporal stability of the HDL-32E is first analysed as the motivation for developing a new, automated calibration method. This is followed by a detailed description of the calibration method that is driven by a novel segmentation method for extracting vertical cylindrical features from the Velodyne point clouds. The proposed segmentation method utilizes the Velodyne point cloud's slice-like nature and first decomposes the point clouds into 2D layers. Then the layers are treated as 2D images and are processed with the Generalized Hough Transform which extracts the points distributed in circular patterns from the point cloud layers. Subsequently, the vertical cylindrical features can be readily extracted from the whole point clouds based on the previously extracted points. The points are passed to the calibration that estimates the cylinder parameters and the LiDAR's additional parameters simultaneously by constraining the segmented points to fit to the cylindrical geometric model in such a way the weighted sum of the adjustment residuals are minimized. The proposed calibration is highly automatic and this allows end users to obtain the time-variant additional parameters instantly and frequently whenever there are vertical cylindrical features presenting in scenes. The methods were verified with two different real datasets, and the results suggest that up to $78.43 \%$ accuracy improvement for the HDL-32E can be achieved using the proposed calibration method.
\end{abstract}

\section{INTRODUCTION}

The Velodyne LiDAR series has been adopted into many unmanned robotics and mobile mapping systems (MMSs) since 2006 due to its unique merits such as its high data capture capacity. The Velodyne HDL-32E was launched in 2011 as an ultra-compact and cost-effective optical sensor for many unmanned robotic vehicles. For example, the HDL-32E has been installed in the Centaur 2 and KRex robotic rovers (Pedersen et al., 2012) developed by the National Aeronautics and Space Administration (NASA) in the United States for lunar survey. Clearpath Robotics Inc. in Canada uses the HDL$32 \mathrm{E}$ in their robotic vehicles for obstacle detections (Clearpath, 2013). On the other hand, the HDL-32E is also widely used by the mobile mapping communities, for example: the Mandli MMS (Mandli, 2013) developed by Mandli Communications Inc., the ScanLook MMS by the LiDAR USA Inc. (LiDAR USA, 2013), and the VISAT ${ }^{\mathrm{TM}}$ system (VISAT, 2013) jointly developed by the University of Calgary and its industrial partners. Due to its compact size, the HDL-32E can be also readily installed on a backpack to form a hand-held MMS (Glennie et al., 2013).

The error modelling and calibration of the Velodyne series (e.g. Muhammad and Lacroix, 2010; Glennie and Lichti 2011; Atanacio-Jiménez et al., 2011; Chen and Chien, 2012; Glennie,
2012) has been intensively studied during the past several years. A more detailed discussion of these studies can be found in Chan and Lichti (2013). More recently, Glennie et al. (2013), and Chan and Lichti (2013) investigated in situ self-calibration of the Velodyne HDL-32E, and their error recovery results are promising. However, the self-calibration procedures might not be very straightforward for some end users and they mostly involve manual selection of many reference features. Thus, a simple and quick calibration method which requires the least amount of manual work is desired by users. Apart from this, due to the strongly time-variant nature of the Velodyne measurement, the self-calibration method may not be the best solution for reducing the error, there is a need for another type of calibration method.

This paper proposes an automatic calibration method for the Velodyne LiDAR based on vertical cylindrical features, and is demonstrated with the Velodyne HDL-32E. This method is particularly suitable for those users whose primary interest is only the LiDAR's intrinsic parameters or additional parameters (APs), rather than the exterior orientation parameters of the LiDAR, and also for those who need the APs updated frequently. One of the main focuses of this paper is the segmentation of the vertical cylindrical features from the Velodyne point cloud for automating the subsequent calibration. The vertical cylindrical features are used because 
they can be easily found in many indoor environments as structural pillars (Chan and Lichti, 2012), and outdoors as concrete or metallic poles in most road corridors ( $\mathrm{Pu}$ et al., 2011). The temporal stability of the HDL-32E is also analysed in this paper to confirm the need for frequent calibrations, as suggested by Glennie and Lichti (2011) for the HDL-64E S2.

\section{VELODYNE HDL-32E AND ITS MODEL}

The HDL-32E has approximate dimensions of $8.5 \mathrm{~cm} \times 8.5 \mathrm{~cm}$ $\times 15 \mathrm{~cm}(\mathrm{~L} \times \mathrm{W} \times \mathrm{H})$ and consists of a spinning laser emitting/receiving window on the top of a static base. There are 32 individual one-dimensional (1D) laser rangefinders which are radially oriented on a panel (Figure 1). The whole panel rotates about the z-axis continuously when power is connected. It has approximately $41.3^{\circ}$ and $360^{\circ}$ fields of view (FOV) in the vertical and horizontal directions respectively. Its data capture rate is approximately 700000 points/s (Velodyne, 2013).

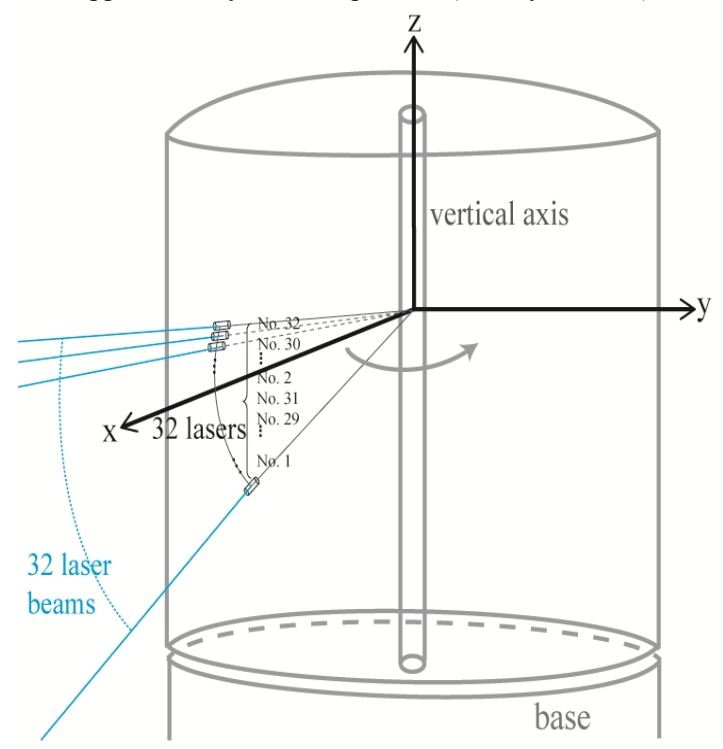

Figure 1. Postulated Velodyne HDL-32E’s configuration

According to the user manual (Velodyne, 2013), the mathematical model of the HDL-32E for point $i$ captured by laser $j$ at scanning position $k$ is given by:

$$
\left(\begin{array}{c}
x_{i j k} \\
y_{i j k} \\
z_{i j k}
\end{array}\right)=\left(\begin{array}{c}
\rho_{i j k} \cos \left(\alpha_{j}\right) \sin \left(\theta_{i j k}\right) \\
\rho_{i j k} \cos \left(\alpha_{j}\right) \cos \left(\theta_{i j k}\right) \\
\rho_{i j k} \sin \left(\alpha_{j}\right)
\end{array}\right)
$$

where $\rho$ and $\theta$ are the observations for the range and the horizontal angular position (horizontal angle), respectively, and $\alpha$ is the fixed vertical angle of the laser.

\section{TEMPORAL STABILITY ANALYSIS}

In order to examine the temporal stability of the HDL-32E, a 26 $\mathrm{cm} \times 26 \mathrm{~cm}$ Spectralon target with $99 \%$ reflectance was placed approximately $2 \mathrm{~m}$ away from the scanner to collect measurements $(\rho$ and $\theta$ ) for a $9000 \mathrm{~s}$ period (Figure 2). The LiDAR system was inclined several times in order to capture all 32 lasers' measurement to the target. There was a $1800 \mathrm{~s}$ rest period in between acquisition periods for any two inclination settings. The range measurements of the sixteen upper lasers (those having positive nominal vertical angles and even numbers denoted by the manufacturer) between the scanner and the centre of the target within a small angular window $\left(0.5^{\circ}\right)$ were averaged and plotted in Figures 3 and 4.
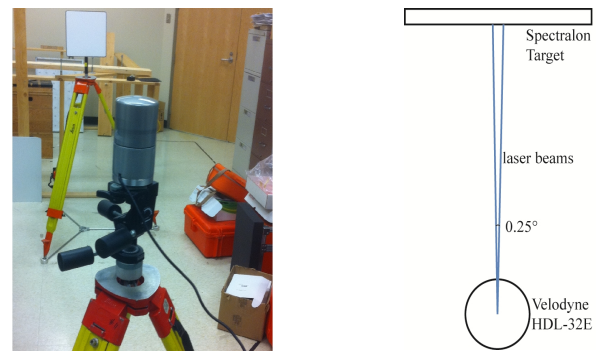

Figure 2. The experimental setup (left) and its diagram on the xy-plane (right) for temporal stability analysis

As can be seen from Figures 3 and 4, none of the lasers has stable range measurements over the $9000 \mathrm{~s}$ period. Some of them fluctuate by more than $0.05 \mathrm{~m}$, which is at least 3 times greater than the thickness $(1.42 \mathrm{~cm})$ of the Spectralon, target assembly. Moreover, an approximate warm-up time of $2000 \mathrm{~s}$ was found for most lasers.

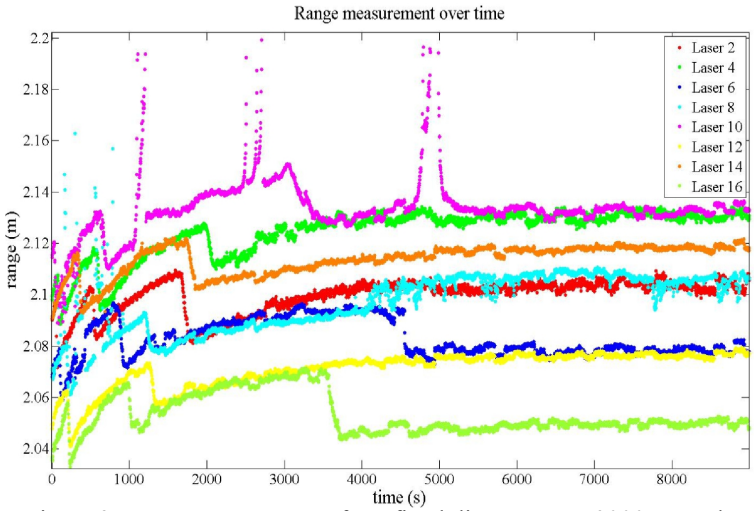

Figure 3. Range measurement for a fixed distance over 9000 seconds (Lasers: 2, 4, 6, 8, 10, 12, 14 and 16)

A short subset of data $(60 \mathrm{~s})$ after the first $5000 \mathrm{~s}$ from five of the lasers was analyzed more closely (Figure 5). It can be seen that all the lasers' measurements vary with magnitudes of several millimetres over the time, and up to $9 \mathrm{~mm}$ range difference was observed for one of the lasers. Consequently, the self-calibration method might not be the best solution for recovering such time-varying range errors. A more straightforward calibration to enable frequent recovery of the measurement distortion should therefore be developed.

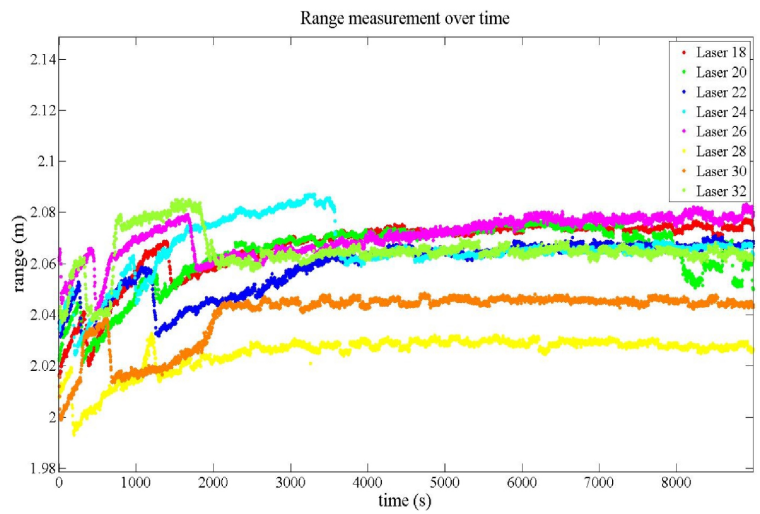

Figure 4. Range measurement for a fixed distance over 9000 seconds (Lasers: 18, 20, 22, 24, 26, 28, 30 and 32) 


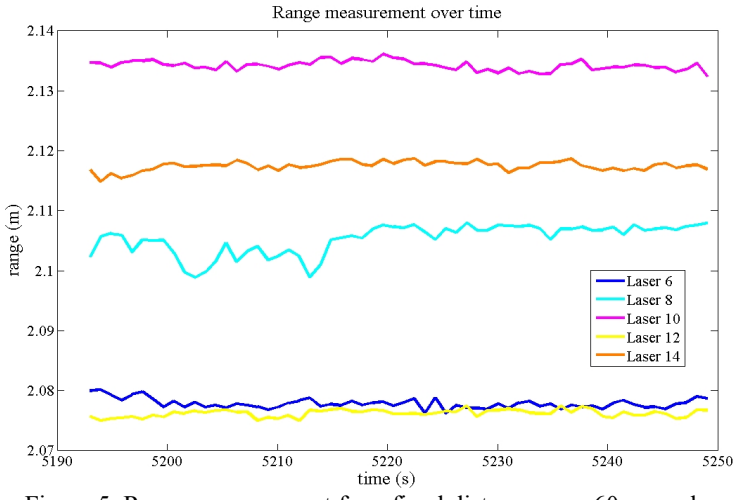

Figure 5. Range measurement for a fixed distance over 60 seconds (Lasers: 6, 8, 10,12 and 14)

\section{PROPOSED METHODOLOGY}

\subsection{Cylinder segmentation}

Similar to Luo and Wang (2008), Lehtomäki et al. (2010) and $\mathrm{Pu}$ et al. (2011), the proposed segmentation method recognizes multiple slices of the cylindrical objects. The multiple slice decomposition concept is particularly efficient for the Velodyne point clouds as they are composed of slices due to the fixed radial orientation of the individual lasers.

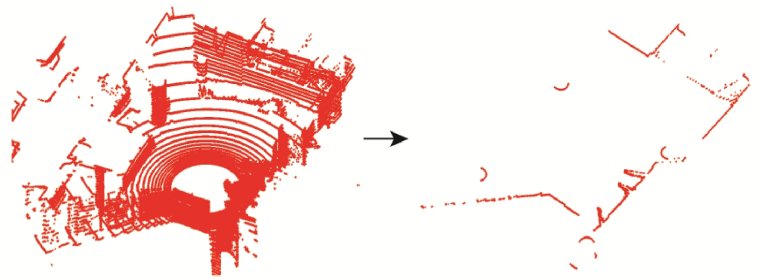

(a) The whole Velodyne point cloud (red)

(b) Layer and density segmentation

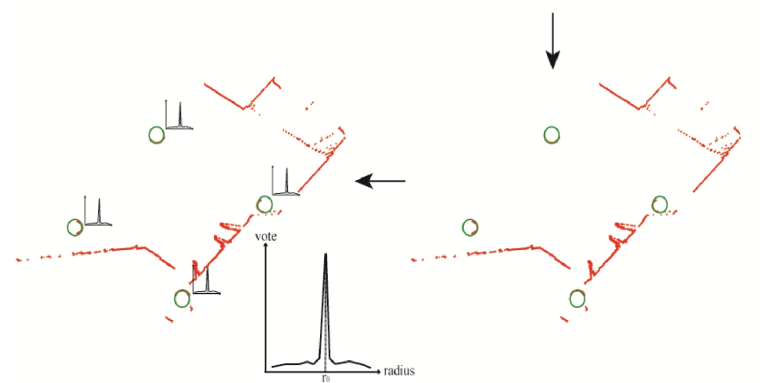

(d) Radius histogram examination

(c) 2D Hough circle detection (green)
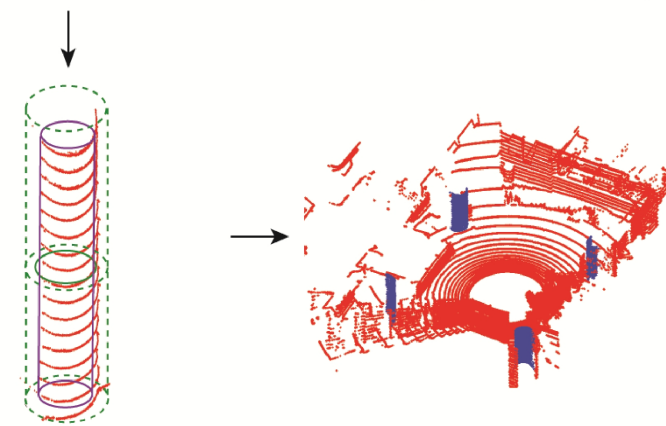

(e) Cylinder extraction (green dash)

followed by cylinder fitting with blunder

(f) The segmented cylinders (blue) detection (megenta)

Figure 6. The proposed vertical cylinder segmentation process

The cylindrical objects consist of multiple circular slices which can be detected using various methods. In our approach, the Generalized Hough Transform (GHT; Ballard, 1981) is used.
The GHT is robust to incompleteness and distortion of the target shapes and the presence of noise. All of these conditions exist in uncorrected Velodyne point cloud slices.

Cylindrical features in point clouds can be detected by directly transforming the whole point cloud into a five dimensional (5D) Hough space, but this would be quite inefficient in terms of computational power and memory consumption. Rabbani and van den Heuvel (2005) broke down the 5D space into two subspaces (a 2D space for the cylinder rotations and a 3D space for the centre and the radius) in order to enhance the search efficiency for detecting cylinders from point clouds. However, their approach would not be as straightforward as the slicebased Hough circle detection method which is a nearly-2D search method (with input of the approximate radius, otherwise, it is purely $3 \mathrm{D}$ ).

\subsubsection{Segmentation process}

Figure 6 shows the proposed cylinder segmentation process. First, the Velodyne point cloud that possesses some vertical pillars is computed from the raw measurements (Figure 6a). This is followed by the extraction of the middle layer of the point cloud using the vertical angles and point density segmentation based on the magnitude of the range measurement (Figure 6b). Then, the point cloud layer is treated as an edge image. The cylinders will appear as arcs which will be detected by the GHT as shown in Figure 6c. An approximate value(s) of the radius (radii) of the pillars is/are needed in order to reduce the search space of the transform. A vote threshold is also needed.

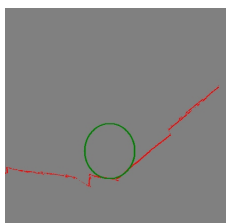

Figure 7. The over-segmented circle (green) by the GHT from the point cloud of a wall corner (red)

After the circle recognition, the radius histogram check method (Ioannou et al., 1999) is applied (Figure 6d) for each circle to check if they are over-segmented by the GHT. Sometimes corner regions between two high-density walls as shown in Figure 7 might be treated as a circle by the GHT. The radius histogram check basically counts the distance between each point to the centre. If it is a circular feature there will be a sharp peak at the radius of the circle. In this work, the peak threshold was set to be $90 \%$ within a radius window $\left(\mathrm{r}_{0} \pm 3 \mathrm{~cm}\right)$. Finally, the extracted circles with an added buffer (the dashed green lines in Figure 6e) in the middle layer are projected to the other layers to form a 3D cylinder window segmenting the cylinder point cloud. This is followed by least-squares fitting to estimate the cylinder parameters and also to remove blunders. The vertical cylinder model (f) for cylinder $q$ is given as

$$
f\left(x_{\mathrm{c}_{q}}, y_{\mathrm{c}_{q}}, \omega_{q}, \phi_{q}, r_{q}\right)=x_{i j k}^{\prime 2}+y_{i j k}^{\prime 2}-r_{q}^{2}
$$

$$
\left(\begin{array}{c}
x_{i j k}^{\prime} \\
y_{i j k}^{\prime} \\
z_{i j k}^{\prime}
\end{array}\right)=\mathbf{R}_{\mathbf{2}}\left(\phi_{q}\right) \mathbf{R}_{\mathbf{1}}\left(\omega_{q}\right)\left(\begin{array}{c}
x_{i j k}-x_{c_{q}} \\
y_{i j k}-y_{c_{q}} \\
z_{i j k}
\end{array}\right)
$$


where $\left(x_{\mathrm{c}}, y_{\mathrm{c}}\right)$ are the centre coordinates of the cylinder in the $x$ $y$ plane, $\omega$ and $\phi$ are the tilt angles of the cylinder about the $x$ axis and $y$-axis respectively, and $r$ is the radius of the cylinder. The final, blunder-free cylinders in the scene are then segmented (Figure 6f) and are ready to be input into the calibration described in the Section 4.1.

\subsection{Cylinder-based calibration}

The segmented cylinders are used as the input for the proposed calibration method, which is somewhat analogous to plumb-line calibration (Brown, 1971) for cameras. Instead of fitting 2D image co-ordinates to straight lines, the measured 3D point coordinates, augmented with the APs, are constrained to fit to the cylindrical objects according to the weighted least-squares criterion. For $m$ cylinders used to calibrate $n$ lasers, the calibration parameter vector is

$$
\vec{X}=\left[\begin{array}{lllll}
\vec{x}_{1}^{\mathrm{T}} & \ldots & \vec{x}_{m}^{\mathrm{T}} & \vec{x}_{\Delta \rho}^{\mathrm{T}} & \vec{x}_{\Delta \theta}^{\mathrm{T}}
\end{array}\right]^{\mathrm{T}}
$$

where $\vec{x}_{q}=\left[\begin{array}{lllll}x_{c} & y_{c} & \omega & \phi & r\end{array}\right]_{q}^{\mathrm{T}}$ is the parameter vector for cylinder $q, \quad \vec{x}_{\Delta \rho}=\left[\begin{array}{lll}\Delta \rho_{1} & \cdots & \Delta \rho_{n}\end{array}\right]^{\mathrm{T}}$ and $\vec{x}_{\Delta \theta}=\left[\begin{array}{lll}\Delta \theta_{1} & \cdots & \Delta \theta_{n}\end{array}\right]^{\mathrm{T}}$ are the vectors of the rangefinder offsets and horizontal angle offsets respectively. The functional model for point $i$ with raw observations for laser $j$ at position $k$, having the observation vector $\vec{l}_{i j k}=\left[\begin{array}{ll}\rho_{i j k} & \theta_{i j k}\end{array}\right]^{\mathrm{T}}$ which lies on the surface of cylinder $q$ is given by:

$$
\begin{gathered}
f\left(\vec{l}_{i j k}, \vec{x}_{q}, \Delta \rho_{j}, \Delta \theta_{j}\right)=x_{i j k}^{\prime 2}+y_{i j k}^{\prime 2}-r_{q}^{2} \\
\left(\begin{array}{l}
x_{i j k}^{\prime} \\
y_{i j k}^{\prime} \\
z_{i j k}^{\prime}
\end{array}\right)=\mathbf{R}_{2}\left(\phi_{q}\right) \mathbf{R}_{\mathbf{1}}\left(\omega_{q}\right)\left(\begin{array}{c}
\left(\rho_{i j k}-\Delta \rho_{j}\right) \cos \left(\alpha_{j}\right) \sin \left(\theta_{i j k}-\Delta \theta_{j}\right)-x_{c_{q}} \\
\left.\rho_{i j k}-\Delta \rho_{j}\right) \cos \left(\alpha_{j}\right) \cos \left(\theta_{i j k}-\Delta \theta_{j}\right)-y_{c_{q}} \\
\left(\rho_{i j k}-\Delta \rho_{j}\right) \sin \left(\alpha_{j}\right)
\end{array}\right)
\end{gathered}
$$

Unlike in self-calibration, the scanner EOPs are not estimated and no a priori EOP information is needed. The method just uses the point clouds of several vertical cylindrical objects (preferably evenly distributed to provide a stronger network) for estimating the APs. The vertical angles are not estimated in the proposed calibration as preliminary testing revealed that they are highly correlated to the rangefinder offset. Moreover, the vertical angles are less time dependent as they are mounting parameters.

\section{EXPERIMENT}

Two data sets were captured by the same HDL-32E at two different locations at the University of Calgary campus (Figures $8 \mathrm{a}$ and $8 \mathrm{~b}$ ). Both the scenes have several large concrete pillars (with radii approximately equal 40 and $50 \mathrm{~cm}$ respectively). All the point clouds of the pillars ( 4 vertical pillars for each dataset) were successfully segmented using the proposed method. The extracted cylinders from each second of data over a 10 second period (Dataset 1 and 2) were then passed to the proposed calibration.

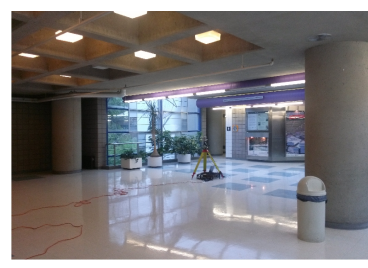

(a)

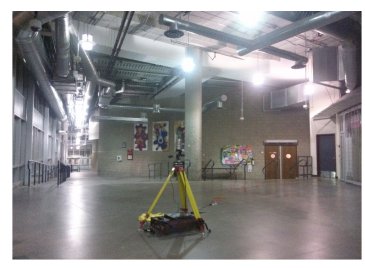

(b)
Figure 8. Two scenes contain vertical pillars at University of Calgary (a) For Dataset 1 (b) For Dataset 2

\section{RESULT ANALYSIS}

\subsection{Estimated parameters}

\subsubsection{Estimated rangefinder offsets $(\Delta \rho)$}

Figures 9 and 10 show the estimated $\Delta \rho$ and the corresponding standard deviations for the calibration using the segmented cylinders from Dataset 1 , respectively. It can be seen that $\Delta \rho$ can vary from several millimetres to $1.5 \mathrm{~cm}$ (Laser 32) over a 10 s period.

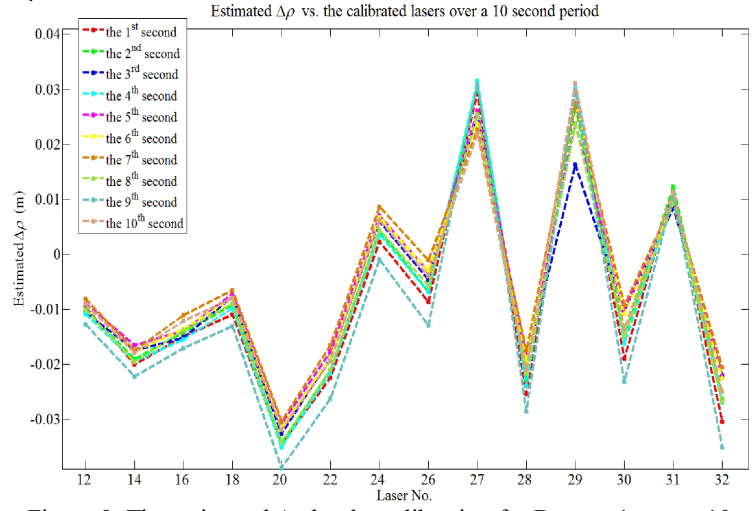

Figure 9 . The estimated $\Delta \rho$ by the calibration for Dataset 1 over a 10 second period

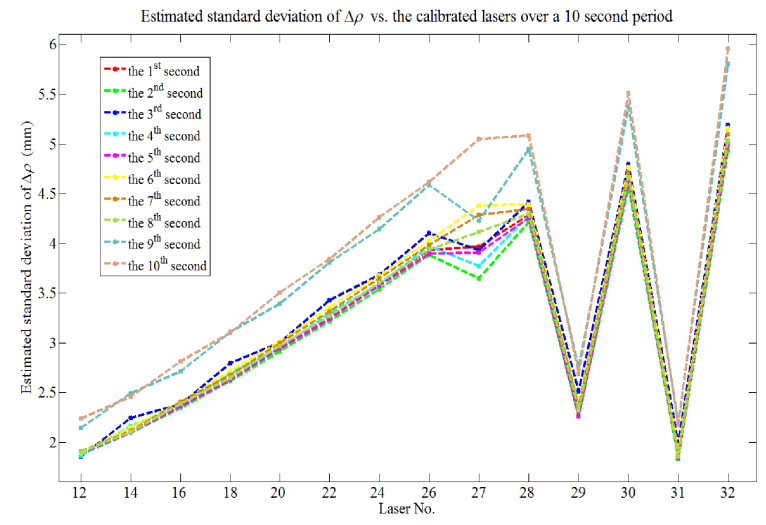

Figure 10. The standard deviations of the estimated $\Delta \rho$ by the calibration for Dataset 1 over a 10 second period

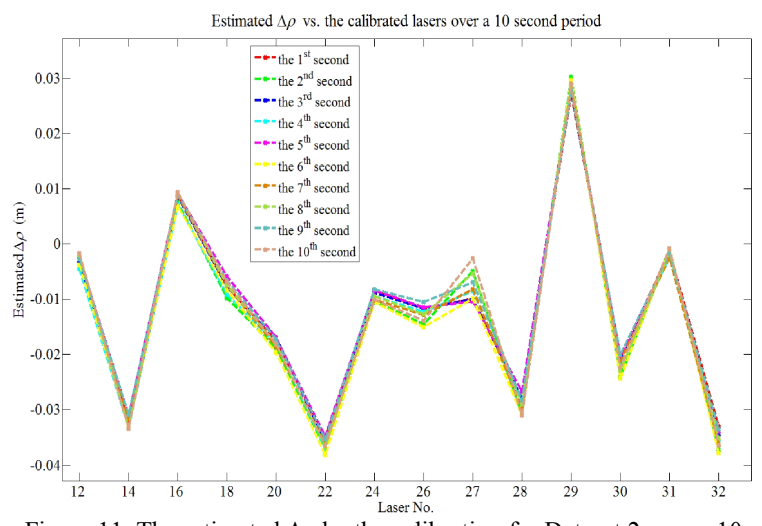

Figure 11. The estimated $\Delta \rho$ by the calibration for Dataset 2 over a 10 second period

As can be seen from Figure 10, the lasers at lower vertical angles generally have lower standard deviations. This can be attributed to the fact that these lasers collect relatively shorter range measurements. Thus, they have higher precisions. The apparently lower precisions in the $9^{\text {th }}$ and $10^{\text {th }}$ seconds are due 
to more erroneous measurements at these two moments, which is consistent with the highest magnitudes of $\Delta \rho$ found in Figure 10. Similarly, Figures 11 and 12 respectively show $\Delta \rho$ and the standard deviations of the Dataset 2 calibration. The deviation of $\Delta \rho$ over the $10 \mathrm{~s}$ period is not as significant as that of Dataset 1 but it can reach up to $0.8 \mathrm{~cm}$ (Laser 27).

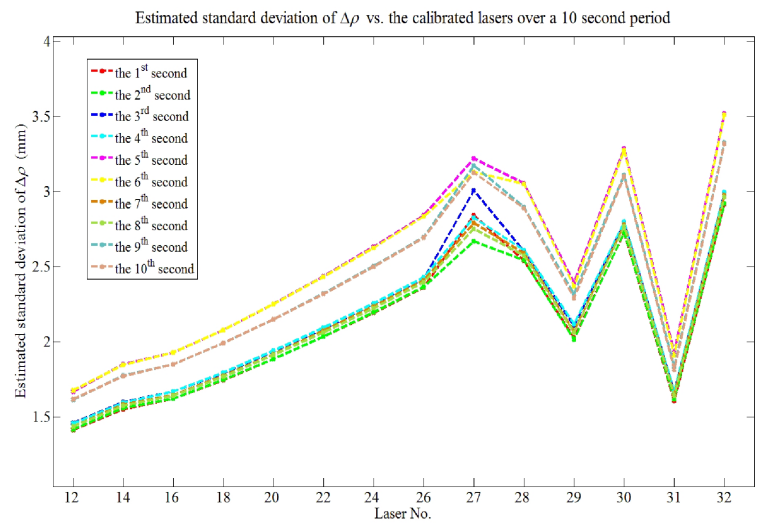

Figure 12. The standard deviations of the estimated $\Delta \rho$ by the calibration for Dataset 2 over a 10 second period

\subsubsection{Estimated horizontal angle offsets $(\Delta \theta)$}

The group of the estimated calibration parameters, $\Delta \theta$, is shown in Figure 13 and the standard deviations are shown in Figure 14 for the calibration for Dataset 1 . The deviations of $\Delta \theta$ in the 10 second period are as significant as $\Delta \rho$ described in Section 6.1.1., reaching up to $0.12^{\circ}$ (Laser 32). The precisions for the $9^{\text {th }}$ and $10^{\text {th }}$ seconds are the lowest as found for $\Delta \rho$ in Figure 10.

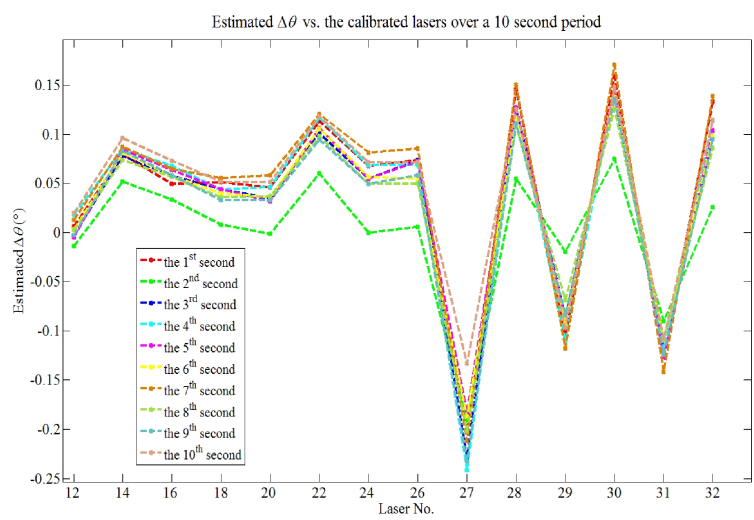

Figure 13. The estimated $\Delta \theta$ by the calibration for Dataset 1 over a 10 second period

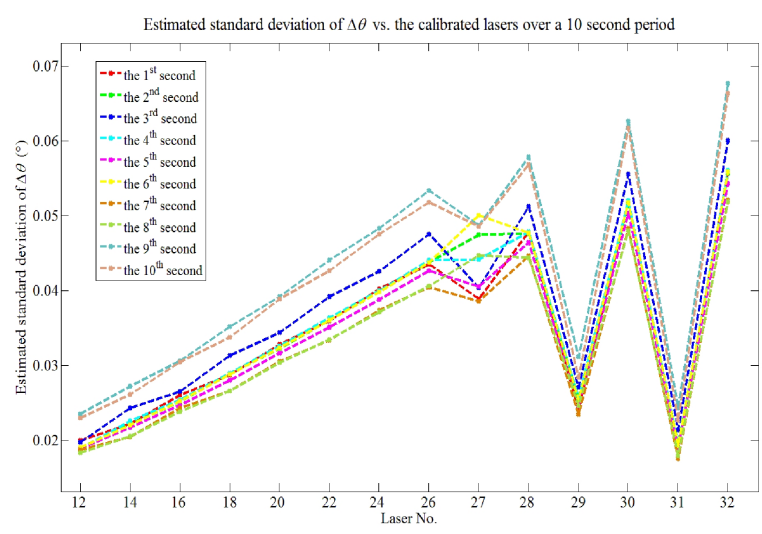

Figure 14. The standard deviations of the estimated $\Delta \theta$ by the calibration for Dataset 1 over a 10 second period
Figures 15 and 16 show the estimated $\Delta \theta$ and the standard deviations for Dataset 2 respectively. In Figure 15, an approximately $0.14^{\circ}$ deviation (Laser 32) can be found among $\Delta \theta$ over the period. This indicates that the deviation is as significant as that shown in Figure 13 for Dataset 1. A very similar trend can be found on the precisions shown in Figures 14 and 16 for the two datasets.

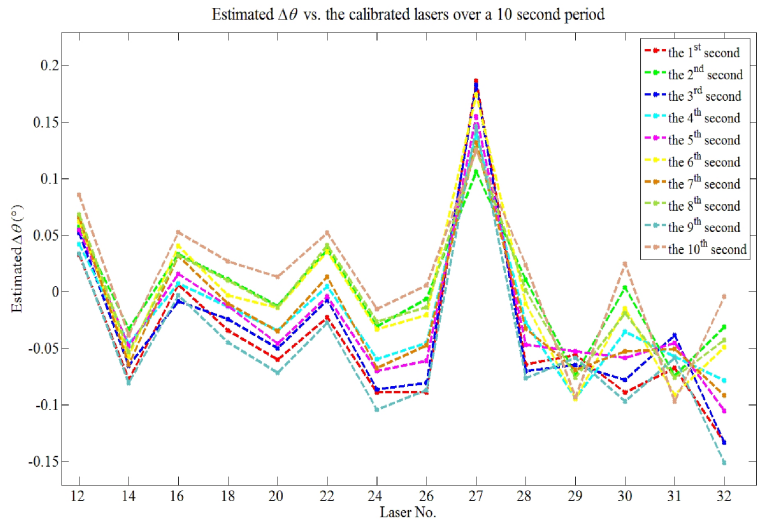

Figure 15. The estimated $\Delta \theta$ by the calibration for Dataset 2 over a 10 second period

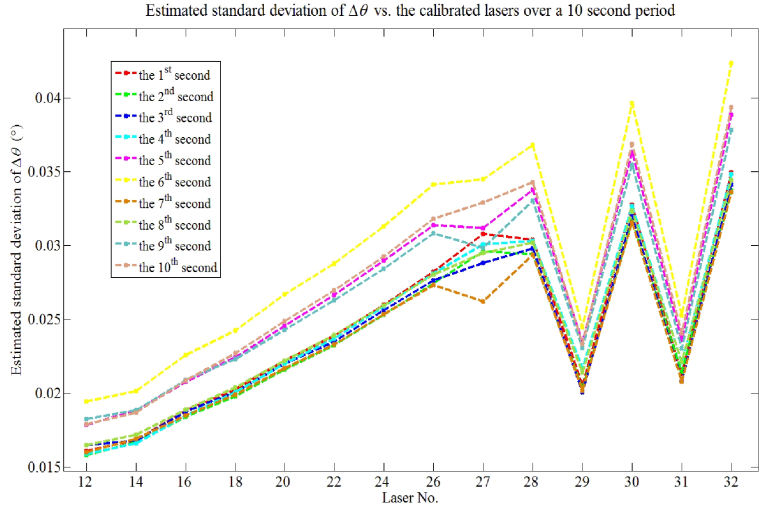

Figure 16. The standard deviations of the estimated $\Delta \theta$ by the calibration on Dataset 2 over a 10 second period

\subsection{Reconstructed point cloud accuracy}

The ultimate goal of calibration is to find accurate APs that can compensate the systematic errors in point clouds. Thus, the estimated parameters were used to reconstruct the point cloud of several check planes which are outside the calibration zone in Datasets 1 and 2 and they were then examined by least-squares fitting.

Table 1. The RMS of the misclosures associated with lasers having the largest improvement rate of the check plane fitting

\begin{tabular}{|c|c|c|c|c|c|c|}
\hline & \multicolumn{3}{|c|}{ Dataset 1 } & \multicolumn{3}{c|}{ Dataset 2 } \\
\hline $\begin{array}{c}\text { Second } \\
(\mathrm{s})\end{array}$ & $\begin{array}{c}\text { Before } \\
\text { Calibration } \\
(\mathrm{m})\end{array}$ & $\begin{array}{c}\text { After } \\
\text { Calibration } \\
(\mathrm{m})\end{array}$ & $\begin{array}{c}\text { Bmprovement } \\
(\%)\end{array}$ & $\begin{array}{c}\text { Before } \\
\text { Calibration } \\
(\mathrm{m})\end{array}$ & $\begin{array}{c}\text { After } \\
\text { Calibration } \\
(\mathrm{m})\end{array}$ & $\begin{array}{c}\text { Improvement } \\
(\%)\end{array}$ \\
\hline 1 & 0.0220 & 0.0074 & 66.32 & 0.0172 & 0.0055 & 67.95 \\
\hline 2 & 0.0220 & 0.0074 & 66.32 & 0.0272 & 0.0066 & 75.55 \\
\hline 3 & 0.0238 & 0.0077 & 67.47 & 0.0180 & 0.0059 & 67.31 \\
\hline 4 & 0.0238 & 0.0077 & 67.47 & 0.0299 & 0.0076 & 74.49 \\
\hline 5 & 0.0232 & 0.0085 & 63.21 & 0.0179 & 0.0060 & 66.41 \\
\hline 6 & 0.0217 & 0.0078 & 63.98 & 0.0276 & 0.0060 & 78.43 \\
\hline 7 & 0.0248 & 0.0084 & 66.10 & 0.0190 & 0.0068 & 64.29 \\
\hline 8 & 0.0248 & 0.0084 & 66.10 & 0.0250 & 0.0076 & 69.46 \\
\hline 9 & 0.0254 & 0.0083 & 67.48 & 0.0189 & 0.0060 & 68.32 \\
\hline 10 & 0.0254 & 0.0083 & 67.48 & 0.0273 & 0.0071 & 74.13 \\
\hline
\end{tabular}

The accuracies of the all the reconstructed check planes were improved after the calibration. The root mean square (RMS) values of the planar misclosures associated with lasers having 
the largest improvement rate for one of the check planes over the $10 \mathrm{~s}$ period is shown in Table 1. It was found that up to 2.2 $\mathrm{cm}$ improvement was achieved. The improvement rates are very significant, reaching up to $78.43 \%$.

\section{CONCLUSIONS}

In this paper, the temporal stability of the range measurements of the Velodyne HDL-32 was first discussed to provide the motivation for the development of a straightforward, on-site calibration that allows end users to readily find the APs. We proposed an automatic calibration method that is driven by a Hough Transform based cylinder segmentation method that utilizes the slice-like nature of the Velodyne point cloud. The proposed calibration method is highly automatic and it makes the frequent calibration (in every 1 second interval) of the Velodyne LiDAR become possible. The calibration results are also shown to be promising as up to $78.43 \%$ improvement in check plane misclosure was realized for the calibrated system. The concepts developed in this paper can be extended to the Velodyne LiDAR operating in kinematic mode in which outdoor roadside pole-like objects such as lamp poles can be segmented and used for frequent, time-dependent calibration.

\section{ACKNOWLEDGEMENTS}

The authors wish to acknowledge Tecterra Inc. in Canada for providing both financial and technical support. The first author would also like to express his gratitude to the Antje Graupe Pryor Foundation estimated by Mr. Werner Graupe for providing the Werner Graupe International Fellowship in Engineering to support his study and research.

\section{REFERENCES}

Atanacio-Jiménez, G., Hurtado-Ramos, J.B. and GonzálezBarbosa, R., 2011. LIDAR Velodyne HDL-64E calibration using pattern planes. International Journal of Advanced Robotic Systems, Vol. 8, No. 5, pp.70-82.

Ballard, D.H., 1981. Generalizing the Hough Transform to detect arbitrary shapes. Pattern Recognition, Vol.13, No.2, p.111-122.

Brown D. C., 1971. Close-range camera calibration. Photogrammetric Engineering, Vol. 37, No. 8, pp. 855-866.

Chan, T. O. and Lichti, D. D., 2012. Cylinder-based selfcalibration of a panoramic terrestrial laser scanner. The International Archives of the Photogrammetry, Remote Sensing and Spatial Information Sciences, 39 (Part B5), 169-174.

Chan, T. O. and Lichti, D. D., 2013. Feature-based selfcalibration of Velodyne HDL-32E LiDAR for terrestrial mobile mapping applications. The $8^{\text {th }}$ International Symposium on Mobile Mapping Technology, Tainan, Taiwan, $1^{\text {st }}-3^{\text {rd }}$ May, 2013. (On CD-Rom)

Chen, C.-Y. and Chien, H.-J., 2012. On-Site sensor recalibration of a spinning multi-beam LiDAR system using automatically-detected planar targets. Sensors, 12(10), pp.13736-13752.

Clearpath, 2013. Clearpath Robotics Inc. http://www.clearpa throbotics.com/grizzly/ (Accessed 29 May 2013).
Glennie, C., 2012. Calibration and kinematic analysis of the Velodyne HDL-64E S2 LiDAR sensor. Photogrammetric Engineering and Remote Sensing, 78(4), pp.1-9.

Glennie, C., Brooks, B., Ericksen, T., Hauser, D., Hudnut, K., Foster, J. and Avery, J., 2013. Compact multipurpose mobile laser scanning system - initial tests and results. Remote Sens., 5 (2), pp. 521-538.

Glennie, C. and Lichti, D.D., 2011. Temporal stability of the Velodyne HDL-64E S2 scanner for high accuracy scanning applications. Remote Sens., 3, pp. 539-553.

Ioannou, D., W. Duda and F. Laine, 1999. Circle recognition through a 2D Hough Transform and radius histogramming. Image and Vision. Computing, 17, pp. 15-26.

Lehtomäki, M., Jaakkola, A., Hyyppä, J., Kukko, A. and Kaartinen, H., 2010. Detection of vertical pole-like objects in a road environment using vehicle-based laser scanning data. Remote Sensing, Vol.2, pp.641-664.

LiDAR USA, 2013. The ScanLook System Specification, http://lidarusa.com/images/ScanLookSpecification.pdf (Accessed 29 Feb 2013).

Luo, D. and Wang, Y., 2008. Rapid extracting pillars by slicing point clouds. International Archives of Photogrammetry, Remote Sensing and Spatial Information Sciences 37 (Part B3), pp. 215-218.

Mandli, 2013. Mandli Communication Inc., project info, http://www.mandli.com/documents/dbi_press.pdf (Accessed 29 Feb 2013).

Muhammad, N. and Lacroix, S., 2010. Calibration of a rotating multi-beam LiDAR. 2010 In Proceedings of the IEEE/RSJ International Conference on Intelligent Robots and Systems, Toulouse, France, pp. 5648-5653.

Pedersen L., Allan M., Utz H., Deans M., Bouyssounouse X., Choi Y., Flückiger L., Lee S. Y., To V., Loh J., Bluethmann W., Burridge R. R., Graf J. and Hambüchen K., 2012. Teleoperated lunar rover navigation using LiDAR. NASA Ames Research Center; Johnson Space Center, Report No. ARC-EDAA-TN5509, Available Online:

http://ntrs.nasa.gov/archive/nasa/casi.ntrs.nasa.gov/2012001684 9_2012017669.pdf (Accessed 29 May 2013).

$\mathrm{Pu}, \mathrm{S} .$, Rutzinger, M., Vosselman, G. and Oude Elberink, S.J., 2011. Recognizing basic structures from mobile laser scanning data for road inventory studies. ISPRS Journal of Photogrammetry and Remote Sensing, 66 (2011) 6 pp. 28-39.

Rabbani, T. and van den Heuvel, F.A., 2005b. Efficient hough transform for automatic detection of cylinders in point clouds. International Archives of the Photogrammetry, Remote Sensing and Spatial Information Sciences, 36 (Part 3/W19) pp. 60-65.

Velodyne 2013.Velodyne HDL-32E User's Manual, http://velodynelidar.com/lidar/products/manual/639113\%20HDL-32E\%20manual_Rev\%20E_NOV2012.pdf (Accessed 11 Jun 2013).

VISAT, 2013. VISATTM Van 2006, http://www.amsvisat.com/VVan/documents/vvan2006 letter_lo wres.pdf (Accessed 11 Jun 2013). 